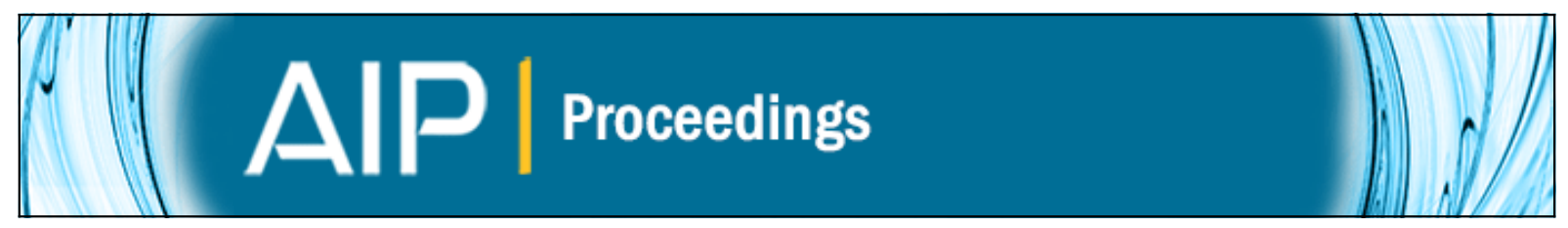

\title{
Far field deposition of scoured regolith resulting from lunar landings
}

A. B. Morris, D. B. Goldstein, P. L. Varghese, and L. M. Trafton

Citation: AIP Conference Proceedings 1501, 1220 (2012); doi: 10.1063/1.4769681

View online: http://dx.doi.org/10.1063/1.4769681

View Table of Contents: http://scitation.aip.org/content/aip/proceeding/aipcp/1501?ver=pdfcov

Published by the AIP Publishing

Articles you may be interested in

Direct numerical simulation of the near-field dynamics of annular gas-liquid two-phase jets

Phys. Fluids 21, 042103 (2009); 10.1063/1.3112740

A Vibrofluidized Reactor for Resource Extraction from Lunar Regolith

AIP Conf. Proc. 813, 1101 (2006); 10.1063/1.2169291

Numerical study of liquid-hydrogen droplet generation from a vibrating orifice

Phys. Fluids 17, 082103 (2005); 10.1063/1.1946767

DSMC and Navier-Stokes Study of Backflow for Nozzle Plumes Expanding into Vacuum

AIP Conf. Proc. 762, 355 (2005); 10.1063/1.1941562

Challenges of three-dimensional modeling of microscale propulsion devices with the DSMC method AIP Conf. Proc. 585, 464 (2001); 10.1063/1.1407597 


\title{
Far Field Deposition of Scoured Regolith Resulting from Lunar Landings
}

\author{
A. B. Morris ${ }^{\mathrm{a}}$, D. B. Goldstein ${ }^{\mathrm{a}}$, P. L. Varghese ${ }^{\mathrm{a}}$, and L. M. Trafton ${ }^{\mathrm{b}}$ \\ ${ }^{a}$ The University of Texas at Austin. ASE-EM Dept., 1 University Station, C0600, Austin, TX 78712, USA. \\ ${ }^{b}$ The University of Texas at Austin. Astronomy Dept., 1 University Station, Clmt, Austin, TX 78712, USA.
}

\begin{abstract}
As a lunar lander approaches a dusty surface, the plume from the descent engine impinges on the ground, entraining loose regolith into a high velocity dust spray. Without the inhibition of a background atmosphere, the entrained regolith can travel many kilometers from the landing site. In this work, we simulate the flow field from the throat of the descent engine nozzle to where the dust grains impact the surface many kilometers away. The near field is either continuum or marginally rarefied and is simulated via a loosely coupled hybrid DSMC - Navier Stokes (DPLR) solver. Regions of two-phase and polydisperse granular flows are solved via DSMC. The far field deposition is obtained by using a staged calculation, where the first stages are in the near field where the flow is quasi-steady and the outer stages are unsteady. A realistic landing trajectory is approximated by a set of discrete hovering altitudes which range from $20 \mathrm{~m}$ to $3 \mathrm{~m}$. The dust and gas motions are fully coupled using an interaction model that conserves mass, momentum, and energy statistically and inelastic collisions between dust particles are also accounted for. Simulations of a 4 engine configuration are also examined, and the erosion rates as well as near field particle fluxes are discussed.
\end{abstract}

Keywords: Plume Impingement, Hybrid Continuum-DSMC, Particle-Laden Flows.

PACS: 47.45.Dt, 52.20.Hv, 96.20.Ka, 47.61.Jd, 47.55.Kf

\section{INTRODUCTION}

When a rocket lands on the Moon the engine exhaust plume will strike the lunar surface and disturb and disperse regolith. During the Apollo moon landings, rocket blast sprays of dust were observed to begin at about 40m altitude and completely or nearly completely obscured the landing site for the last 20 to $30 \mathrm{~m}$ of descent requiring the terminal approach to be flown on instruments [1]. Simulations suggest [2,3] that some of the entrained particles may obtain velocities that exceed $1 \mathrm{~km} / \mathrm{s}$. Further, the range of particulate trajectories is very large because the moon does not have a background atmosphere that can impede the dust sprays. These high velocity dust sprays can strike structures such as a lunar outpost or potentially damage the heritage from the Apollo missions. Such debris/dust may be difficult or impossible to clear from surfaces because of electrostatic attractions and the highly adhesive properties of lunar soil. Such issues were recognized during the Apollo era when Apollo 12 landed $163 \mathrm{~m}$ from the Surveyor III spacecraft. The surfaces of the Surveyor III that faced the Apollo 12 LM were found to be heavily pitted and discolored by the plume ejecta [4]. Such issues may arise within the next several years as private companies compete to land a spacecraft safely on the moon and win the Google Lunar X PRIZE. A bonus prize of up to $\$ 4 \mathrm{M}$ will also be awarded if the spacecraft can perform a pinpoint landing near an Apollo heritage site. Accurate modeling of the dust sprays can help ensure safe landing distances such that spacecraft do not sandblast the Apollo hardware.

The flow field associated with a rocket plume impinging on the lunar regolith is complex. Within the descent engine bell, the exhaust flow is hot, chemically reacting, compressible, and largely inviscid. Just outside the nozzle, the plume expands into vacuum and the temperature, pressure and density decrease rapidly while the Mach number becomes very large. If the plume strikes the regolith, shock waves will form nearly parallel to the surface and turn the flow outwards. The outwardly expanding wall-jet is a highly compressible stagnation point flow and a viscous boundary layer develops on the rough lunar surface. The resultant surface stresses may exceed the cohesive stresses of the soil and therefore lift and accelerate loose regolith particles into the wall-jet boundary layer. The entrained regolith has a polydisperse distribution of grain sizes and these particles can collide inelastically with each other. This two-phase flow is typically heavily mass loaded by dust, and consequently the dust particle and gas molecule motions are strongly coupled in the near field. As this two-phase flow expands towards vacuum the gas and dust number densities decrease, and eventually the dust motion will decouple from that of the gas. After decoupling, the aerodynamic forces are negligible and the dust particles travel ballistically. The direct simulation Monte Carlo (DSMC) method is well suited for including such physics and is the primary solver used in the current work.

(C) 2012 American Institute of Physics 978-0-7354-1115-9/\$30.00 


\section{METHOD}

When near the lunar surface, the exhaust gases in the vicinity of the rocket engine are highly collisional and can be simulated using continuum models. However, as the flow expands towards vacuum, continuum assumptions break down and a kinetic model must be used. In this work, we loosely couple the Navier Stokes solver DPLR [5] to a DSMC solver using volume reservoir cells [6]. The interface between the two solvers is drawn within the continuum region and where the interface normal Mach number is supersonic. DPLR is only used to solve for the gas flow inside the rocket nozzle accounting for nozzle geometry effects, and in the near field of the plume expansion, while DSMC is used to simulate the two-phase flows everywhere else. In the current work, we chose to model a descent engine similar to the Lunar Module descent engine (LMDE) because this allows us to compare to certain data from the Apollo landings. The actual LMDE exhaust gases comprised many different species with comparable concentrations, but only the most prevalent species, $\mathrm{H}_{2} \mathrm{O}$, is presently included in our model. Vibrational and rotational energies are included in the DSMC solver and assumed to be in equilibrium in the continuum solver. Near touchdown, the thrust of the descent engine was approximately 3,000lbs and the ISP was 305s [7]. The area ratio of the expander was 47.5 and the diameter at the nozzle exit plane was $1.32 \mathrm{~m}$. The flow is sonic at the nozzle throat and the corresponding density and temperature are computed from isentropic flow relations and are $0.1275 \mathrm{~kg} / \mathrm{m}^{3}$ and $2491 \mathrm{~K}$ respectively. The recovered thrust of the modeled engine is $2960 \mathrm{lbs}$ and the mean exit plane density, velocity, and temperature are $1.18 \times 10^{-3} \mathrm{~kg} / \mathrm{m}^{3}, 2860 \mathrm{~m} / \mathrm{s}$, and $577 \mathrm{~K}$.

\section{Dust Entrainment and Granular Collisions}

The erosion and transport of regolith is handled entirely within the DSMC solver. Metzger has identified three possible erosion mechanisms: viscous erosion, bearing capacity failure, and diffused gas eruption [8]. Viscous erosion is considered the dominant erosion mechanism and occurs when the surface shear exceeds the cohesive strength of the soil causing small dust particles to roll along the surface. The rolling particles can then impact neighboring grains and hop upwards and become entrained. Scaling relationships for erosion rates were empirically determined [9]. These tests show that the erosion rate, $\Phi_{e}$, scales with the densimetric Froude number.

$$
\Phi_{e} \propto \frac{\rho_{g} U_{g}^{2}}{2 g \rho_{p} D_{p}} \equiv \mathrm{Fr}^{2} .
$$

The left hand side of Eq. 1 represents the particle mass flux, $\rho_{g} U_{g}^{2} / 2$ is the dynamic pressure of the free stream gas outside of the viscous boundary layer, $\rho_{p}$ is the density of an individual dust grain, $g$ is the acceleration of gravity, and $D_{p}$ is the mean diameter of the dust grains. The dynamic pressure is evaluated at a distance slightly above the boundary layer, $5 \mathrm{~cm}$ in this work, because the surface roughness scales are assumed to be large relative to the boundary layer scale. The scaling constant is selected such that the simulated crater depth is comparable to what was observed throughout the Apollo missions; a shallow 2-4cm deep crater beneath the descent engine.

In our framework, dust particles are treated as special representative particles with an assumed temperature and mass and diameter that are many orders of magnitude greater than those for gas molecules. The dust particle's temperature is representative of the energy stored within the grain's crystalline lattice and is not the same as the granular or macroscopic temperature. The highest particle temperatures do not exceed $1000 \mathrm{~K}$ and phase change energy is neglected. The aerodynamic diameter distribution has been measured for the fine and ultrafine portion of lunar regolith [10] and we approximate this distribution with a discrete set of $200 \mathrm{~nm}, 800 \mathrm{~nm}, 2 \mu \mathrm{m}, 5 \mu \mathrm{m}, 8 \mu \mathrm{m}$, and $11 \mu \mathrm{m}$ grains. The particle size distribution of eroded grains is assumed to be the same as the undisturbed regolith. A fully coupled gas-particle model is used [11]; as momentum and energy are transferred to the dust grains, momentum and energy are removed from the gas to ensure conservation. In this model, the computed drag and heat transfer to the dust particles is valid for any arbitrary non-equilibrium gas flow where the grain $K n, K n \equiv \lambda_{g} / D_{p}$, is large. The drag and energy transfer to the dust particles are computed by assuming that gas molecules that collide with a particle have some combination of diffuse and specular scattering, but gas molecules are assumed to undergo purely diffuse reflection in our work. One could directly simulate this in DSMC by colliding gas molecules with each dust grain, but for larger particles this is not feasible because the computed number of collisions becomes impractically large for large cross sections. A drag and energy transfer model which represents the effect of many collisions is used instead [12]. The gas is coupled to the dust by performing an additional collision step where selected gas molecules reflect diffusely off a dust particle.

Collisions between dust particles can occur during lunar landings, such as near the erosion regime where there are large velocity gradients and particle number densities, or near symmetry planes between nozzles if a multi- 
engine configuration is used [13]. In addition, the grain number densities can be quite different from each other. Consequently, a species dependent weighting parameter, i.e. the number of real particles represented by each simulator, is used. Granular collisions are modeled similarly for a dilute gas because collisions are infrequent and assumed to occur instantaneously. Unlike gas collisions, inelastic granular collisions are modeled with an assumed restitution coefficient. The coefficient of restitution between colliding grains is in general a function of impact speed, particle geometry, and material properties. The details of the full collision are approximated with a simplified hard sphere model that assumes a constant coefficient of restitution. The normal and tangential coefficients of restitution are both assumed to be 0.8 , and the scattering is isotropic for this special case. The generalized No Time Counter (NTC) method [14] is employed which allows for different particle weights.

A key parameter that affects the acceleration of dust particles is the ratio between the particle cross section and mass. The density of a soil particle, $\rho_{p}$, is defined as the ratio of the particle mass to the volume displaced by the grain and the recommended value is $3100 \mathrm{~kg} \mathrm{~m}^{3}$ [15]. To account for irregular particle shapes, an apparent particle density, $\rho_{a}$, is used to compute the mass of a particle with a known aerodynamic cross section. The apparent density is defined as the ratio between of the particle mass to the volume of a hard sphere with an equivalent cross section. The apparent density can be related to particle density via the volume coefficient, $\alpha_{v}$, which is defined as the volume displaced by the grain divided by the cubed aerodynamic diameter, $d_{a}$. The recommended value for the volume coefficient is 0.34 [15] and the resulting apparent density is $1900 \mathrm{~kg} / \mathrm{m}^{3}$. The aerodynamic cross section is then simply $\pi d_{a}^{2} / 4$.

\section{Far Field Simulations}

The far field is simulated by using a set of staged calculations. This is done because the near field calculations require small cell sizes and a time steps. These are impractically small for simulations of the entire domain for a complete landing. As the solution progresses from the near to far field stages, the time step and cell sizes are increased. The gas and dust flow fields in the near vicinity of the lander can be treated as quasi steady because the flow times are much shorter than the descent rate. In the far field, however, the descent time is comparable or small compared to the ballistic scale times and an unsteady solution is required. A landing trajectory is approximated by a set of discrete hovering altitudes and an assumed descent rate of approximately $0.5 \mathrm{~m} / \mathrm{s}$. The times and altitudes are summarized in Table 1.

TABLE 1. Simulated hovering altitudes and time spent at each altitude.

\begin{tabular}{lc}
\hline Altitude & Duration \\
\hline $20.0 \mathrm{~m}$ & $8 \mathrm{~s}$ \\
$15.0 \mathrm{~m}$ & $6 \mathrm{~s}$ \\
$12.5 \mathrm{~m}, 10.0 \mathrm{~m}, 7.5 \mathrm{~m}$ & $4 \mathrm{~s}$ \\
$5.0 \mathrm{~m}$ & $6 \mathrm{~s}$ \\
$3.0 \mathrm{~m}$ & $8 \mathrm{~s}$ \\
\hline
\end{tabular}

The slowest moving dust particles travel at approximately $300 \mathrm{~m} / \mathrm{s}$ and the flow field is assumed quasi steady at distances within $300 \mathrm{~m}$ of the lander. The near field is simulated using two computational domains for each hovering altitude that extend to 100 and $300 \mathrm{~m}$ respectively. Once steady, the simulators that leave the innermost domain are stored to a patch file that is then used as an input for the next domain. In the second domain, the mean free path is longer and the time step and cell size are increased. Molecules are randomly sampled from the patch file each time step such that the number flux is conserved. Molecules that exit the second domain are similarly stored to a file and used as an input for the first unsteady domain.

The far field is simulated using two unsteady domains that extend to 5 and $500 \mathrm{~km}$ respectively. The first unsteady domain reads the molecules from the steady state patch files starting at $20 \mathrm{~m}$ hovering altitude for $8 \mathrm{~s}$ and then progressing to lower altitudes, Table 1 . The molecules that exit the unsteady domain are stored along with the time at which they exited the domain. These molecules are then read by the second unsteady domain and created at the appropriate simulation time. The unsteady domains are then simulated until all of the dust particles leave the domain or impact the ground.

\section{NUMERICAL RESULTS}

We first present results for a single engine lander descending towards the lunar surface, where the thrust vector is perpendicular to the surface and the flow is axisymmetric. The axisymmetric calculations were performed at the 
Texas Advanced Computing Center and used 48 processors and approximately 2 million cells with 20 million simulators. Next, near field simulations of a hovering 4-engine configuration are presented. The 4-engine configuration was simulated using 360 processors, 65 million cells, and 180 million simulators. A collision cell adaptation scheme is used to redistribute cells to optimize resolution of the local gas mean free path [16].

\section{Single Engine Axisymmetric Simulations}

The spacecraft trajectory for the final $20 \mathrm{~m}$ of descent is approximated by using a discrete sequence of hovering altitudes, $h$. Within the first $300 \mathrm{~m}$ of the lander, the flow is assumed to be quasi-steady for each simulated hovering altitude. The near field gas pressure field is shown, figure 1a, for the rocket engine hovering $3 \mathrm{~m}$ above the surface without the presence of dust. The internal nozzle flow and the near-nozzle plume flow are simulated with DPLR and then coupled to the DSMC solver along the hybrid interface shown. Within the nozzle, a weak compression wave forms near the throat and extends through the nozzle exit plane. This compression wave reflects off the symmetry axis and then interacts with the surface shock, causing the shock standoff distance to be slightly lower near the jet axis. Most of the erosion takes place within the first $30 \mathrm{~m}$ of the jet axis and erosion profiles are shown in Fig. 1b. Roberts developed a model for the surface stresses and erosion in support of the Apollo program [17], and found that the peak stress and thus erosion rate only depends on the engine Mach number and ratio of gas specific heats. From Roberts theory, the location of peak erosion for this simulated engine occurs at $r=0.39 h$. Roberts' theory did not solve for the impinging gas flow field and did not account for real nozzle effects when close to the ground. The location of peak erosion predicted by Roberts' model agrees remarkably well with our simulations at higher altitudes, but over-estimates the location of peak erosion at altitudes below $10 \mathrm{~m}$. This may occur because of nozzle geometry effects.

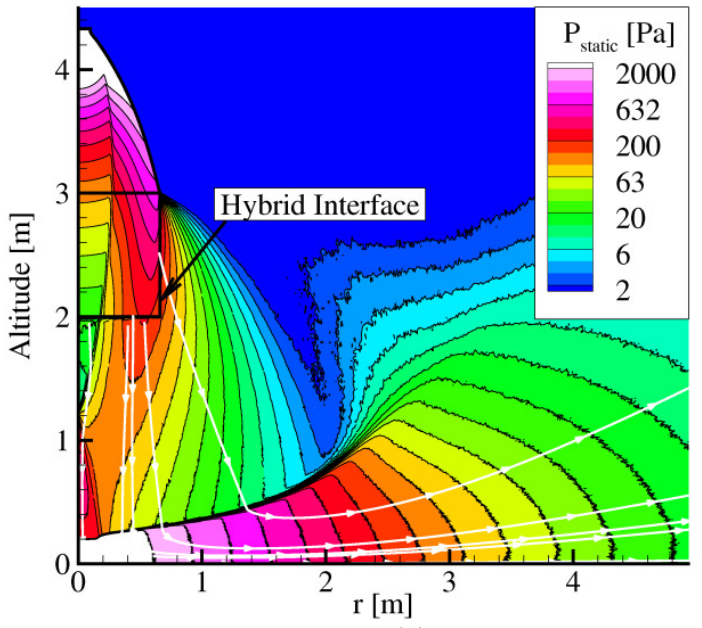

(a)

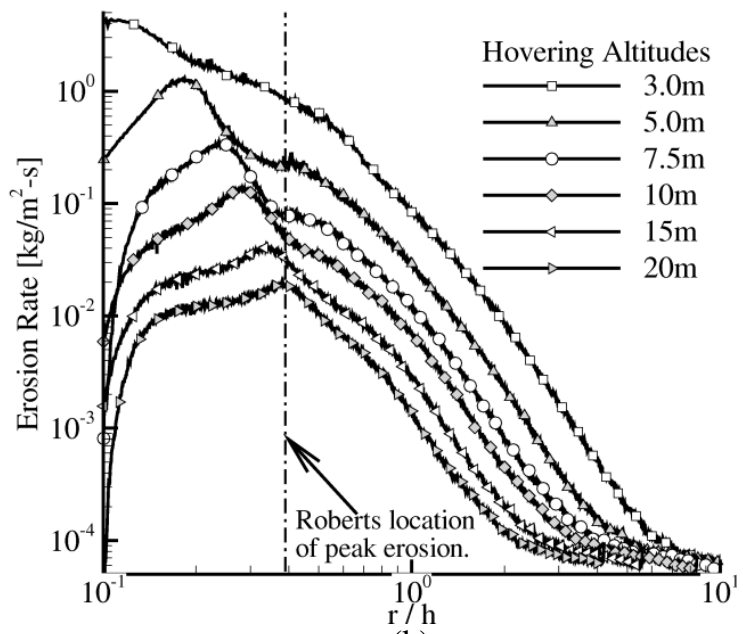

(b)

FIGURE 1. (a) Gas pressure when the engine is hovering $3 \mathrm{~m}$ above the ground. The internal nozzle flow is simulated with DPLR and the hybrid interface is drawn well above the surface shock. (b) Dust erosion profiles for different hovering altitudes.

Although scaling relationships for viscous erosion can be used to estimate the total erosion, they are not sufficient to determine the erosion of specific sized grains in polydisperse mixtures. Therefore, we assume that the particle size distribution of eroded particles is the same as undisturbed regolith. In our model, approximately $41 \%$ of the eroded mass is comprised of the $8 \mu \mathrm{m}$ diameter grains, $27 \%$ from $11 \mu \mathrm{m}$ grains, $25 \%$ from the $5 \mu \mathrm{m}$ grains, and $6 \%$ from the $2 \mu \mathrm{m}$ grains. Smaller 200 and $800 \mathrm{~nm}$ grains are also included, but contain significantly less mass than the other sized particles. In figure 2, the particle mass is plotted versus altitude for different sized grains at various positions for a rocket engine hovering at $5 \mathrm{~m}$. The peak mass flux for all particles decreases proportionally to $r^{-2}$, which is expected for a flow expanding radially outwards. The peak flux is approximately proportional to $r^{-2}$, and values range from $1.6 \mathrm{~g} / \mathrm{m}^{2}$-s at $r=50 \mathrm{~m}, 0.4 \mathrm{~g} / \mathrm{m}^{2}-\mathrm{s}$ at $r=100 \mathrm{~m}$, and $0.1 \mathrm{~g} / \mathrm{m}^{2}-\mathrm{s}$ at $r=200 \mathrm{~m}$. The altitude of peak mass flux occurs at 2.2, 4, and $9 \mathrm{~m}$ for distances 50,100, and $200 \mathrm{~m}$ from the jet axis, respectively. The $8 \mu \mathrm{m}$ particles are the largest contributor to the overall mass flux and this is likely a consequence of the size distribution of eroded particles that we select. 

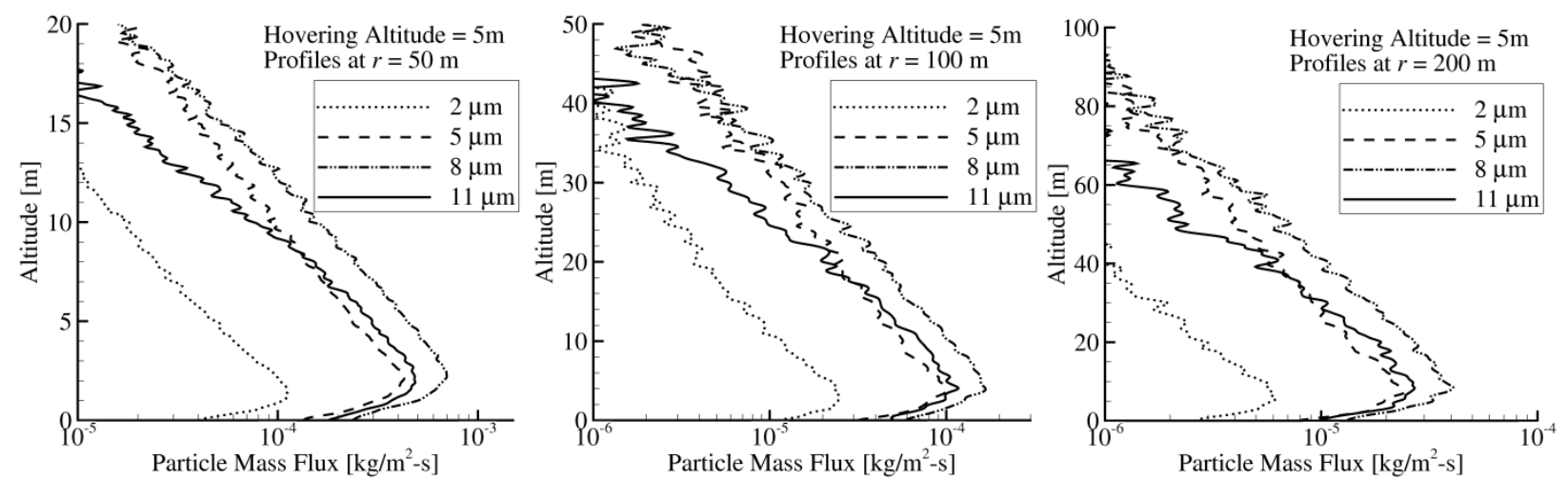

FIGURE 2. Vertical slices of the particle mass flux at various taken 50,100, and $200 \mathrm{~m}$ from the jet axis for a rocket engine hovering at $5 \mathrm{~m}$. Note the logarithmic scale.

The density of the gas decreases as it expands outwards, and eventually the dust particles decouple from the gas. The time scale for the dust to be accelerated to the gas velocity scales linearly with the particle diameter, i.e. the mass of the particle divided by the aerodynamic cross section. The smallest dust particles remain coupled to the gas longer than the larger grains and can reach higher velocities. In figure 3, the relative speed between the dust and gas, referred to as the slip speed, is plotted for $800 \mathrm{~nm}$ and $11 \mu \mathrm{m}$ particles. Regions where there are relatively few dust particles have been blanked for clarity. The slip speeds tend to be larger for the heavier particles, as expected, but there are some qualitative differences between the small and large particles. The $800 \mathrm{~nm}$ particles show an increase in relative velocity near the wall, for distances $r>45 \mathrm{~m}$, despite the growth of the gas boundary layer. This does not occur because of reflection of dust particles off the lunar surface because particles that strike the surface stick. Rather, the increase in slip speed near the wall occurs because of grain-grain collisions between the light particles with the slower moving heavy grains. In general, dust particles tend to track the motion of the gas, but when a granular collision occurs the post-collision velocity will likely not be in same direction as the gas. This causes an increased slip velocity that can persist if the gas and dust are weakly coupled. Further evidence of graingrain collisions is shown near the jet axis, where $800 \mathrm{~nm}$ particles are scattered upwards. These effects are not seen by larger particles because of their relatively large inertia.

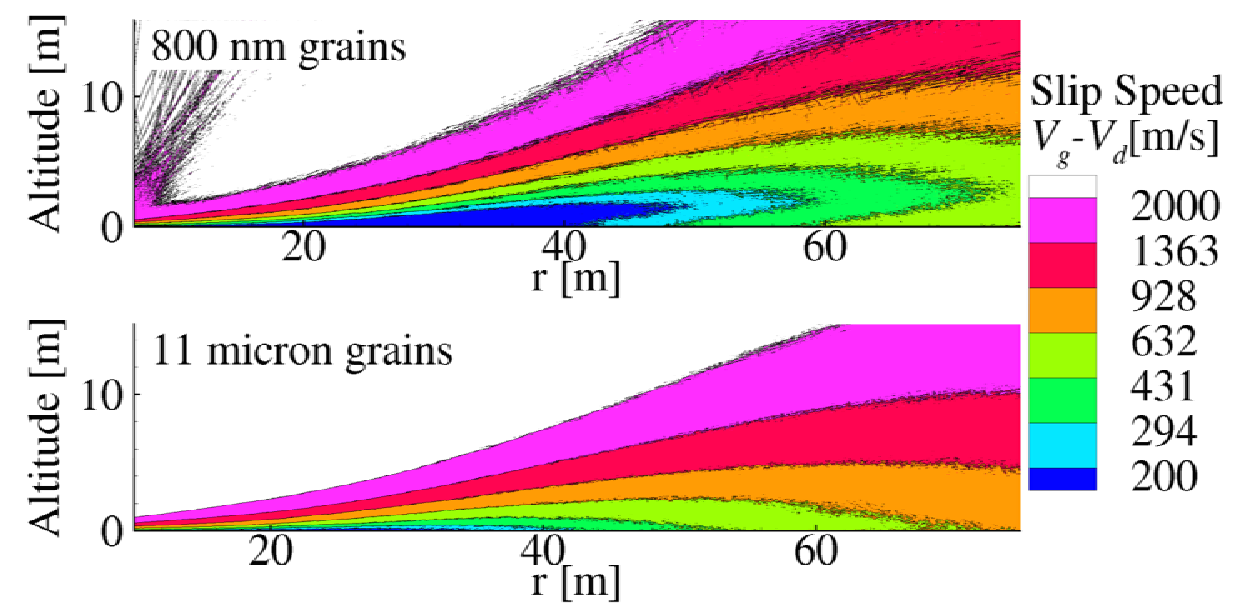

FIGURE 3. Contours of the relative speed between the gas and dust for two different particle sizes.

An unsteady simulation has been performed to see where the scoured dust travels in the far field. At such distances from the lander, the gas flow is free molecular and the dust particles follow ballistic trajectories. In figure 4 , contours of dust number density are presented for $800 \mathrm{~nm}$ and $8 \mu \mathrm{m}$ grains at different times after engine shutoff. The dust cloud diffuses as it travels outwards, but the different sized grains tend to separate. This separation occurs because the smaller particles are able to accelerate to higher velocities, as discussed previously. The smaller $800 \mathrm{~nm}$ particles travel at approximately $1 \mathrm{~km} / \mathrm{s}$ compared to $550 \mathrm{~m} / \mathrm{s}$ for the $8 \mu \mathrm{m}$ particles. 


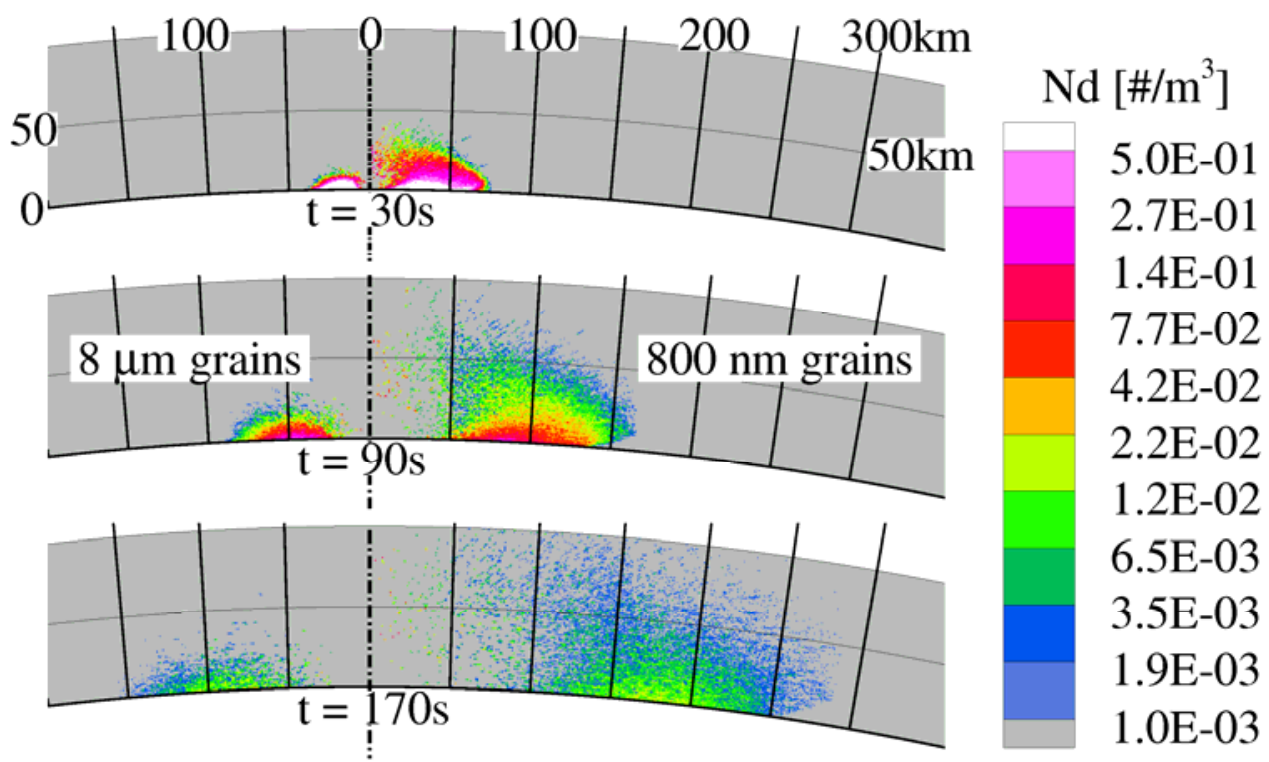

FIGURE 4. Snapshots of $800 \mathrm{~nm}$ (right) and $8 \mu \mathrm{m}$ (left) particle number densities at times 30 , 90, and 170s after engine shutdown. Note that the actual computational domain extends to much higher altitudes and distances from the lander.

The separation of dust particles based on their diameter and velocity results in grain size-dependent deposition patterns. The particles that strike the ground during an entire landing event are counted and the net deposited mass per unit area for different sized grains is shown in figure $5 \mathrm{a}$. Within the first $100 \mathrm{~km}$ of the landing site, the 8 and $11 \mu \mathrm{m}$ grains comprise the bulk of the deposition. Deposition at greater distances from the landing site is dominated by the smaller particles because these particles are accelerated to higher speeds. The flux distribution in figure 5a is then multiplied by the surface cell area to obtain the total deposition as a function of distance from the lander. Particle separation is directly seen by evaluating the distance from the lander at which peak deposition occurs for different sized grains, figure $5 \mathrm{~b}$. Smaller grains are deposited further from the lander and the trend is approximately linear within the $800 \mathrm{~nm}-11 \mu \mathrm{m}$ range.

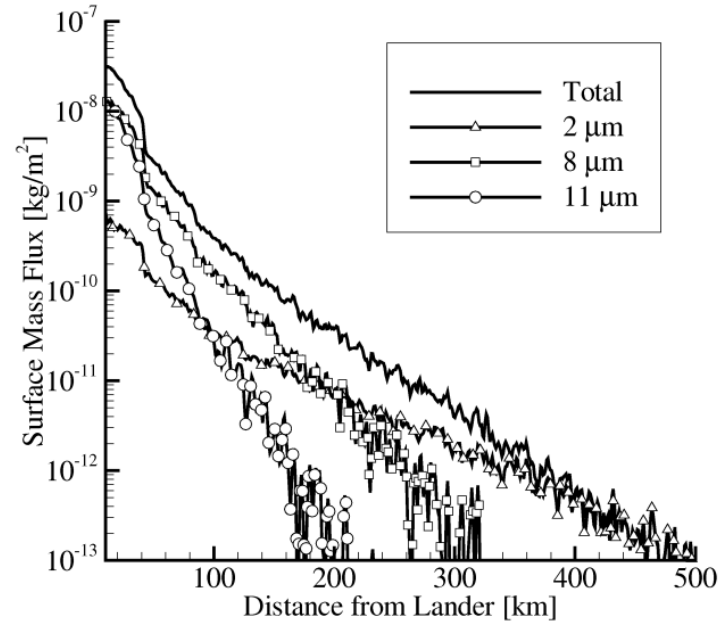

(a)

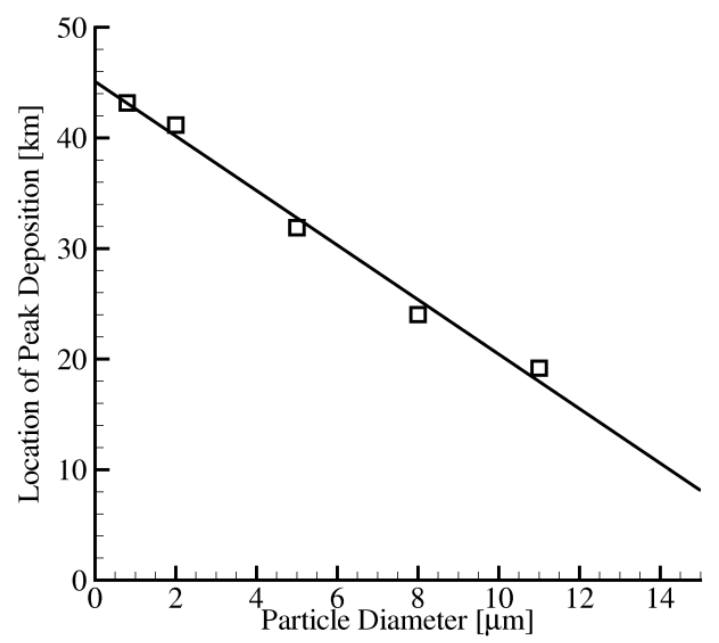

(b)

FIGURE 5. (a) The amount of particle mass deposited per unit area resulting from one landing event. Distributions of specifically sized particles and the overall deposition are plotted versus distance from the landing site. (b) The distance from the lander at which peak deposition occurs for different sized particles. 


\section{Four Engine Configuration}

A multi-engine configuration, where each engine is symmetrically offset by one nozzle diameter is modeled. The mass flow rate of each nozzle is scaled such that the 4 engine configuration has the same net thrust as the single engine simulations discussed above. There are two symmetry planes in this configuration; one slices through the center of the nozzle and the other bisects two adjacent nozzles. These symmetry planes are used to reduce the computational cost, and simulators that strike these boundaries are specularly reflected. In figure $6 \mathrm{a}$, slices of $8 \mu \mathrm{m}$ particle flux are drawn over an isosurface of gas density. An oblique shock forms near the symmetry axis as a result of plume-plume interactions. Below this shock, a large recirculation bubble containing gas forms. Several dust particles are entrained by this vortex and can travel upwards along the symmetry axis, possibly impinging on the lander. Plumes from adjacent nozzles interact with each other, and the dust sprays along this symmetry plane become elevated, as compared to the single nozzle configuration. The peak $8 \mu \mathrm{m}$ particle mass flux at $r=15 \mathrm{~m}$ is $0.02 \mathrm{~kg} / \mathrm{m}^{2}-\mathrm{s}$ and is approximately 2.5 times larger than the corresponding flux for a single engine configuration. Most of the ejecta tend to concentrate near the nozzle symmetry plane while a deficit of dust is observed in the middle of the domain between the two symmetry planes.

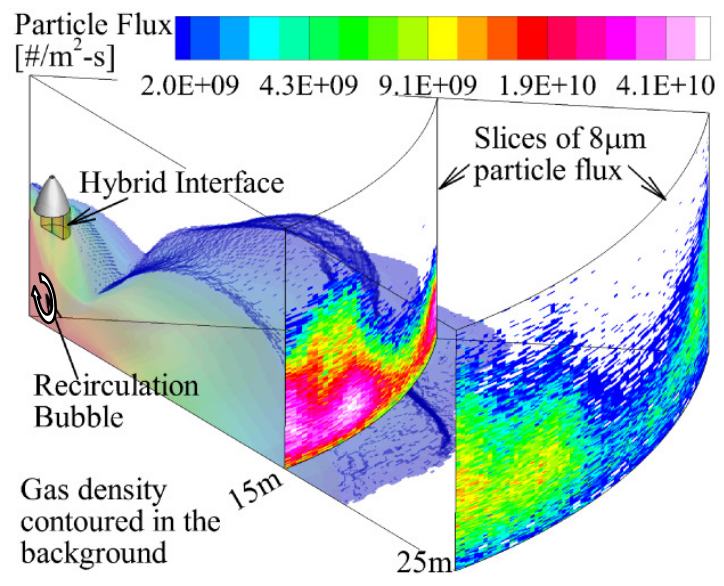

(a)

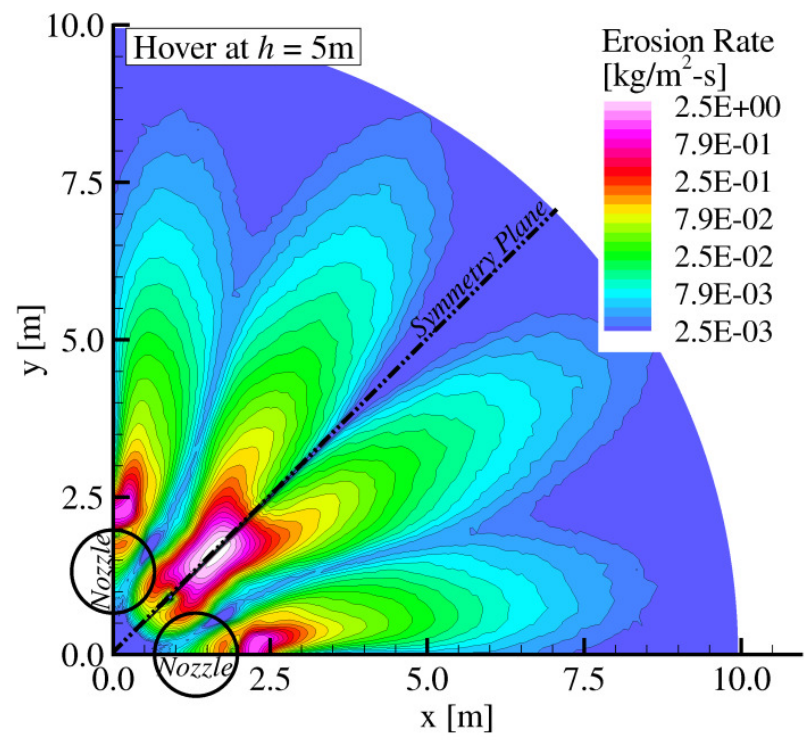

(b)

FIGURE 6. (a) Slices of $8 \mu \mathrm{m}$ particle flux are drawn at distances 15 and $25 \mathrm{~m}$ from the symmetry axis. Contours of gas density are drawn in the background, and one of the four nozzles is drawn for clarity in this octant. (b) Contours of the erosion rate shown relative to two of the nozzles in a quadrant. Note the sample grid is much coarser than the collision grid.

The surface erosion rate is contoured in figure $6 \mathrm{~b}$. The peak erosion occurs near the symmetry plane between adjacent nozzles and is approximately twice that of the single engine case, see figure $1 \mathrm{~b}$. This erosion rate $(\sim 1 \mathrm{~mm} / \mathrm{s})$, however, is insufficient to scour a large trench during a typical landing. The gas dynamic pressure, and thus erosion rate, is reduced at further distances along the marked symmetry plane. This occurs because the gas is coupled to the dust motion, and gas momentum is lost as the dust particles accelerate. The maximum erosion occurs near the symmetry plane between adjacent nozzles, but the region of highest particle flux occurs near the nozzle symmetry plane. The reason for this remains unknown, but this phenomenon was not observed in earlier work when grain-grain collisions were neglected.

\section{CONCLUSIONS}

The impingement of a rocket exhaust plume and the erosion and transport of regolith has been modeled for an entire landing event. The erosion of dust is modeled using empirically derived scaling relationships and the twophase transport is simulated with a fully coupled gas-particle model. A set of discrete particle sizes is used to approximate the particle size distribution of lunar regolith and the effects of inelastic grain-grain collisions are included. We simulate a typical landing by approximating the trajectory by a set of discrete hovering altitudes and 
hovering times. For each hovering altitude, near field quasi-steady solutions are obtained. A staging scheme is then used to patch the quasi-steady solutions into an unsteady far-field domain.

Dust particle fluxes are also shown at different positions when the lander hovers at $5 \mathrm{~m}$. The peak particle flux decreases further from the lander and is approximately proportional to $r^{-2}$. Although not shown in this paper, the particle flux is sensitive to the coefficient of restitution between colliding grains. Granular collisions with large coefficients of restitution tend to cause more diffusion of the dust sheet and thus a lower peak particle flux. The actual coefficient of restitution between colliding grains is not yet known but is a necessary parameter for more accurate modeling.

In the far field, larger particles tend to decouple from the gas earlier and attain lower terminal velocities than the smaller particles. Consequently, as the dust cloud expands to the far field different sized particles tend to separate. The largest particles are deposited closer to the lander than the smaller particles, and for the particle sizes investigated the location of max particle deposition is approximately linearly proportional to the particle diameter. At $50 \mathrm{~km}$ from the landing site, the particle deposition rate is quite small, approximately $10^{-8} \mathrm{~kg} / \mathrm{m}^{2}-\mathrm{s}$.

A 4-engine configuration with the same total thrust as the single engine case has been simulated and near field results are presented. The dust particle flux is highest along the two symmetry planes and is deflected upwards slightly more than the single engine case. The peak particle flux is approximately 2.5 times greater than that for the single engine case. The maximum erosion rates occur near the symmetry planes, and most of the scouring happens along the plane between adjacent nozzles. Although the peak erosion rate is twice as much as the peak erosion for the single engine same thrust case, this is still not sufficient to scour a deep trench.

\section{ACKNOWLEDGMENTS}

This work was graciously funded by NASA grant NNX08AW08G under the LASER program. Simulations were performed on supercomputers maintained by the Texas Advanced Computing Center (TACC).

\section{REFERENCES}

1. S. A. Wagner, "The Apollo Experience Lessons Learned for Constellation Lunar Dust Management", NASA TP-2006213726, Johnson Space Center. (2006).

2. A. B. Morris, D. B. Goldstein, P. Varghese, L. Trafton, "Plume Impingement on a Dusty Lunar Surface", in Proc. of the 27th Intl. Symp. on Rarefied Gas Dynamics, 1333, pp 1187-1192. (2011).

3. J. E. Lane, P. Metzger, C. Immer, X. Li, "Lagrangian Trajectory Modeling of Lunar Dust Particles", in Conf Proc. Earth and Space 2008, Long Beach, CA (2008).

4. C. D. Immer, J. Lane, P. Metzger, S. Clements, Icarus 211, 1089-1102. (2011).

5. M. J. Wright, G. Candler, D. Bose, AIAA Journal 36,1603-1609. (1998).

6. B. D. Stewart, E. Pierazzo, D. Goldstein, P. Varghese, L. Trafton, Icarus 215, 1-16. (2011)

7. W. Hammock, E. Currie, A. Fisher, "Apollo Experience Report: Descent Propusion System", NASA Technical Note D-7143, (1973).

8. P. T. Metzger, J. Smith, J. Lane, J. Geophys. Res. 116, E06005 (2011).

9. P. T. Metzger, J. Lane, C. Immer, J Gamsky, W. Hauslein, X. Li, R. Latta III, C. Donaue, "Scaling of Erosion Rate in Subsonic Jet Experiments and Apollo Lunar Module Landings", Conf Proc. Earth and Space 2010, Honolulu, HI, (2010).

10. P. S. Greenberg, D. Chen, S. Smith, "Aerosol Measurements of the Fine and Ultrafine Particle Content of Lunar Regolith", NASA/TM-2007-214956, Glenn Research Center. (2007).

11. J. M. Burt and I. D. Boyd, "Development of a Two-Way Coupled Model for Two Phase Rarefied Plume Flows", Conf. Proc. of the 42nd AIAA Aerospace Sciences Meeting, Reno, NV. (2004).

12. M. A. Gallis, J. Torczynski, D. Rader, Phys. Fluids 13, 3482-3492 (2001).

13. J. E. Lane, P. Metzger, B. Vu, " Trajectory Modeling of Particles Propelled by a Multi-Engine Rocket Plume on the Lunar Surface", Conf Proc. Earth and Space 2012, Pasadena, CA, (2012).

14. D. P. Schmidt and C. J. Rutland, J. Comp. Phys. 164, 62-80 (2000).

15. G. Heiken, D. Vaniman, B. French, Lunar Sourcebook: A User's Guide to the Moon, New York, Cambridge University Press. (1991).

16. C. H. Moore, "Monte Carlo Simulation of the Jovian Plasma Torus Interaction with Io's Atmosphere and the Resultant Aurora During Eclipse", Ph.D. Thesis, The University of Texas at Austin, 2011.

17. L. Roberts, "The Interaction of a Rocket Exhaust With the Lunar Surface", The Fluid Dynamics Aspects of Space Flight, AGARDograph 87, Vol. II, Gordon \& Breach, 269-290, (1966). 